\title{
A genetic approach to understanding inner ear function
}

James F. Battey, Jr.

National Institute on Deafness and Other Communication Disorders, NIH Building 31, Room 3C02, 31 Convent Drive MSC 2320, 9000 Rockville Pike, Bethesda, Maryland 20892, USA. Phone: (301) 402-0900; Fax: (301) 402-1590; E-mail: batteyj@nidcd.nih.gov.

Within the temporal bone lies the inner ear, home to both the snail-shaped cochlea, critical for auditory function, and the vestibule, providing critical sensory input for balance. Both organs house sensory hair cells, cellular transducers of either sound waves (auditory) or linear or angular acceleration (vestibular) into electrical impulses. These unique cells have at their apices a bundle of microvilli containing parallel actin filaments referred to as stereocilia, whose deflection by as little as a nanometer results in the opening of mechanicallygated ion channels.

In the cochlea, stereocilia are bathed by endolymph, an extracellular fluid with unusually high potassium concentration. When the stereocilia are deflected, potassium from the endolymph flows through transducer channels in the hair cells, resulting in depolarization. Following depolarization, inner hair cells release neurotransmitter at synapses with afferent neurons, while outer hair cells undergo an oscillating change in length that both increases sensitivity and fine tunes responses to sound frequencies. This electrochemical gradient between endolymph and hair cells is thought to require the active, ATP-dependent recycling of potassium from the depolarized hair cells back into the endolymph. The discovery that hearing impairment can result from mutations in genes whose products are essential to this putative recycling pathway suggests that potassium recycling is required for normal auditory function.

Given the remarkable sensitivity and specificity of auditory function, it is not surprising that mutations in hundreds of different genes cause profound and progressive hearing impairment in children (for reviews, see refs. 1-3). Roughly one child in two thousand is born with hearing impairment that may compromise development of normal language skills. About $30 \%$ of these individuals have syndromic hereditary hearing impairment, where other clinical fea- tures are observed. About 70\% have nonsyndromic hearing impairment, where abnormal auditory function is the only obvious clinical outcome resulting from gene mutation. Hundreds of different syndromes have hearing impairment as a cardinal feature, and over sixty genetic loci are known to harbor a gene whose mutation results in nonsyndromic hearing impairment that is inherited in a mendelian fashion. These numbers continue to grow at a rapid rate, indicating that there may be as many as a five hundred different gene products whose function is essential for hearing.

\section{Genetic dissection of potassium recycling in the cochlea}

Studies of hereditary hearing impairment have allowed the identification of many critical, and previously unknown, molecular components of the auditory system, including some that appear to define the unique biological pathway of potassium recycling. Mutations in the potassium channel gene $K C N Q 4$ result in a dominant form of progressive hearing loss (4); this channel is postulated to be of importance in transporting potassium ions out of hair cells. Upon leaving the hair cells, potassium ions are postulated to pass through a network of gap junctions between supporting cells and fibrocytes in the cochlea, ending up in the epithelial marginal cells of the stria vascularis, the structure that secretes potassium-rich endolymph. Connexin 26 and connexin 31 are protein components of these gap junctions. Mutations in the GJB2 gene, encoding connexin 26, are the most common cause of hereditary hearing impairment in Americans, accounting for about one fourth of autosomal recessive hearing impairment (5) and a much higher percentage in other populations $(6,7)$. A sodiumpotassium-chloride cotransporter protein encoded by Slc2a2 helps to pump potassium into the marginal cells that secrete endolymph. Mice with mutations in this gene fail to produce endolymph, resulting in profound hearing loss (8). Completing the postulated recycling loop, channels at the secreting surface of the marginal cells are made up of subunits that are encoded by the KvLQT1 and ISK genes. In humans, mutations in KvLQT1 or ISK result in Jervell and Lange-Nielsen syndrome, a disorder characterized by autosomal recessive congenital hearing impairment and a long QT interval on electrocardiograms (9). Mice engineered to lack a functional isk gene cannot produce normal endolymph and show profound hearing impairment (10).

In this issue of the JCI, Lee et al. (11) report that inactivation of the KvLQT1 gene results in profound hearing impairment and circling behavior characteristic of vestibular abnormality. This mouse model for Jervell and LangeNielsen syndrome recapitulates hearing impairment and manifests a more severe vestibular abnormality, but fails to demonstrate the electrocardiographic abnormalities observed in humans. Histopathologic examination of the inner ear of these mice reveals severe anatomic disruption of the cochlear and vestibular end organs, providing additional insight into the auditory and vestibular abnormalities found in the mouse model and in patients with Jervell and Lange-Nielsen syndrome.

\section{Mouse models for hereditary hearing impairment are essential} These and many other elegant studies underscore the importance of mouse models in furthering understanding of human genetic disease and gene function. When a mutated human gene is mapped to a chromosomal locus, a mouse model with a mutated gene in the corresponding region of the mouse genome can be very helpful in identifying the mutated gene. The power of this approach is amply demonstrated by the effort to clone a deafness gene at the DFNB3 locus, where a mouse model, the shaker-2 mouse, was available. The 
mutated gene was determined by identifying the gene that would rescue normal hearing in the shaker-2 mouse (12). Once the gene (myo15) was found in the mouse, human MYO15 was rapidly cloned and mutations were identified in families with hereditary deafness mapping to the DFNB3 locus (13). The shaker-2 mouse was used for morphology studies at the ultrastructural level, which showed a striking loss of normal hair cell stereocilia, the presumptive cause of hearing loss. Without an animal model for hereditary hearing impairment, it is difficult if not impossible to determine the anatomical, biochemical, and cellular basis for the phenotype, or to use gene rescue to prove unequivocally that the disease-causing gene has been identified. Indeed, when a mouse model does not already exist, it is often necessary to create one as the first step in understanding how the gene mutation results in hearing impairment.

Hundreds of additional mouse models for hearing and vestibular defects will emerge in the next few years as a result of systematic efforts to introduce mutations at random into sperm at high rates. After mutagenesis, the progeny will be screened for auditory and vestibular abnormalities, as well as a host of other phenotypes of biomedical interest. Successful efforts of this type are already under way in the United Kingdom and Germany, and have just begun in the United States in several centers supported by collaborations between institutes within the NIH. It is not unreasonable to speculate that within the next five years there may be a mouse model available for most if not all genes, or combination of genes, whose mutations result in nonsyndromic hearing impairment. This collection of mouse models would be a remarkable resource for identifying the corresponding genes in humans, understanding gene function, and dissecting the molecular pathways essential for auditory function in unprecedented detail (for a review of mouse models for hearing loss, see ref. 14).

Other approaches to identify proteins that are critical for auditory function in particular are also bearing fruit. Successful efforts are underway to identify genes selectively expressed in the cochlea, and to determine whether these genes map to chromosomal loci known to harbor one or more hereditary hearing impairment genes. Using this approach, the genes underlying one type of autosomal dominant deafness (DFNA9) (15), as well as one type of autosomal recessive deafness (DFNB9) (16), were identified. More recently, Zheng et al. identified a collection of genes selectively expressed in outer, but not inner, hair cells (17). Outer hair cells function both to amplify and to fine-tune the auditory signal, and they are essential for important hearing outcomes, such as speech recognition. They perform these functions by changing their length in response to depolarization, activating a voltage-dependent motor in the lateral cell membrane that changes the membrane surface area. Inner hair cells do not share this function, but instead release neurotransmitter at synapses with afferent auditory neurons. The motor molecule was identified as a protein called prestin that is abundantly expressed in outer, but not inner, hair cells. Creation of a mouse lacking a functional prestin is in progress and will provide a powerful tool for understanding this aspect of auditory function.

\section{Potential clinical implications}

Within the last four years, the research community has identified nearly twenty genes whose mutation results in nonsyndromic hereditary hearing impairment. One of these genes, GJB2, is responsible for about $25 \%$ of hereditary hearing impairment in Americans, and an even higher percent in certain defined subpopulations $(6,7)$. Knowledge of these important genes will allow development of genetic tests, leading to rapid and precise diagnosis of hereditary hearing impairment. Increased diagnostic precision is particularly important when a child with hearing impairment is born in a family where both parents and all close relatives have normal hearing. In this situation, it is difficult to determine the etiology of hearing impairment in the absence of genetic tests. Knowledge of the etiology is essential to enable informed counseling for the affected individual, as well as his or her parents and other relatives. Greater diagnostic precision may arm clinicians with important clues about other medical problems that accompany syndromic hearing impairment. Future clinical research may uncover new and different ways to improve the development of language and communication skills among individuals with hearing impairment, basing intervention strategies on genetic information. We are entering a new golden age in our approach to hearing loss, both in terms of our fundamental understanding of auditory function and our ability to provide precise diagnosis, accurate prognosis, and optimal intervention for individuals with hereditary hearing impairment.

\section{Acknowledgments}

The author thanks Thomas B. Friedman, Andrew Griffith, Donald Luecke, and Robert Dobie for critical comments. The author also thanks John Ashkenas for editing and providing an opportunity for this Commentary. James F. Battey, Jr. is the Director, National Institute on Deafness and Other Communication Disorders, NIH.

1. Friedman, T., et al. 2000. Modifier genes of hereditary hearing loss. Curr. Opin. Neurobiol. 10:487-493.

2. Morell, R.J. 1999. Recent progress in hereditary hearing loss. Current Opinion in Otolaryngology \& Head and Neck Surgery. 7:259-265.

3. Steel, K.P. 1999. The benefits of recycling. Science. 285:1363-1364

4. Kubisch, C., et al. 1999. KCQN4, a novel potassium channel expressed in sensory outer hair cells, is mutated in dominant deafness. Cell. 96:437-446.

5. Cohn, E.S., et al. 1999. Clinical studies of families with hearing loss due to mutations in the connexin 26 gene (GJB2/DFNB1). Pediatrics. 103:548-550.

6. Estivill, X., et al. 1998. Connexin-26 mutations in sporadic and inherited sensorineural deafness. Lancet. 351:394-398.

7. Morell, R., et al. 1998. Mutations in the connexin 26 gene (GJB2) among Ashkenazi Jews with nonsyndromic recessive deafness. N. Engl. J. Med. 339:1500-1505.

8. Dixon, M.J., et al. 1999. Mutation of the Na-K-Cl co-transporter gene Slc12a2 results in deafness in mice. Hum. Mol. Genet. 8:1579-1584.

9. Neyroud, N., et al. 1997. A novel mutation in the potassium channel gene KvLQT1 causes the Jervell and Lange-Nielsen cardioauditory syndrome. Nat. Genet. 15:186-189.

10. Vetter, D.E., et al. 1996. Inner ear defects induced by null mutation of the isk gene. Neuron. 17:1251-1264.

11. Lee, M.P., et al. 2000. Targeted disruption of the Kvlqt1 gene causes deafness and gastric hyperplasia in mice. J. Clin. Invest. 106:1447-1455.

12. Probst, F.J., et al. 1998. Correction of deafness in shaker-2 mice by an unconventional myosin in a BAC transgene. Science. 280:1444-1447.

13. Wang, A., et al. 1998. Association of unconventional myosin MYO15 mutations with human nonsyndromic deafness DFNB3. Science. 280:1447-1451.

14. Probst, F.J., and Camper, S.A. 1999. The role of mouse mutants in the identification of human hereditary hearing loss genes. Hear. Res. 130:1-6.

15. Robertson, N.G. 1997. Mapping and characterization of a novel cochlear gene in human and in mouse: a positional candidate gene for a deafness disorder, DFNA9. Genomics. 46:345-354.

16. Yasunaga, S., et al. 1999. A mutation in OTOF, encoding otoferlin, a FER-1-like protein, causes DFNB9, a nonsyndromic form of deafness. Nat. Genet. 21:363-369.

17. Zheng, J., et al. 2000. Prestin is the motor protein of cochlear outer hair cells. Nature. 405:149-155. 